\title{
MIXING IN COASTAL AREAS INFERRED FROM LYAPUNOV EXPONENTS: IMPLICATIONS FOR TRANSPORT
}

\author{
Orfila $\mathrm{A}^{1}$, Galan $\mathrm{A}^{2}$, Simarro $\mathrm{G}^{3}$, Sayol J M ${ }^{4}$
}

\begin{abstract}
We study the horizontal surface mixing and the transport induced by waves, using local Lyapunov exponents and high resolution data from numerical simulations of waves and currents. By choosing the proper spatial (temporal) parameters we compute the Finite Size and Finite Time Lyapunov exponents (FSLE and FTLE) focussing on the local stirring and diffusion inferred from the Lagrangian Coherent Structures (LCS). The methodology is tested by deploying a set of eight lagrangian drifters and studying the path followed against LCS derived under current field and waves and currents.
\end{abstract}

Keywords: Mixing; Finite Size Lyapunov Exponents; Lagrangian Coherent Structures

\section{INTRODUCTION}

Transport, dispersion and mixing of coastal waters are of crucial interest due to the ecological and economical importance of these areas. Despite the increasing advance in the scientific description of the physical processes that take place in the coastal ocean a high degree of uncertainty still remains when predicting trajectories of particles advected by the flow in coastal environments. On one hand, coastal dynamics is influenced by deep water conditions over a complex topography and driven at the surface by highly variable (spatially and temporally) wind conditions. On the other hand, the effects of wind generate waves modifying the current field by the excess of momentum flux induced by waves. In summary, flows in coastal areas are the combination of currents with variations of hours or days and wave oscillatory flows with periods of seconds to tens of seconds. This interaction is usually accounted in coastal ocean models by the radiation stress concept (Longuet-Higgins and Stewart, 1964). Besides, mixing properties of passive tracers in time dependent flows, depend on the chaotic nature of the Lagrangian particle trajectories (Lapeyre, 2002).

Trajectory of water particles have been studied extensively under a lagrangian point of view ( $\ddot{z} z g \ddot{o} k$ men, 2000; Molcard et al., 2006; Galan et al., 2012). Moreover, the stretching by advection is usually analyzed by means of the Lyapunov exponents (Haller and Yuan, 2000; Mancho et al., 2008; HernandezCarrasco et al., 2011). Local finite-time Lyapunov exponents are the exponential rate of separation, averaged over infinite time, of fluid parcels separated infinitesimally (d'Ovidio et al., 2004). These lagrangian descriptors provide the existence of patterns that are a proxy of the whole flow (Wiggins, 1992). The resulting structures are known as Lagrangian Coherent Structures (LCS).

Finite Time Lyapunov Exponent (FTLE) for a fluid particle located initially in a position $\mathbf{x}$ at time $t_{0}$ with a finite time integration $T$ is given by,

$$
\lambda^{T}(\mathbf{x}, t)=\frac{1}{|T|} \ln \frac{\delta \mathbf{x}\left(t_{0}+T\right)}{\delta \mathbf{x}\left(t_{0}\right)},
$$

where $\delta \mathbf{x}\left(t_{0}+T\right)$ is the distance between the particle and a neighboring particle advected by the flow after time $T$. Analogously, the Finite Size Lyapunov Exponent (FSLE) for two particles initially separated a distance $\delta_{0}$ is,

$$
\lambda^{\delta_{f}}\left(\mathbf{x}, t, \delta_{0}, \delta_{f}\right)=\frac{1}{|\tau|} \ln \frac{\delta_{f}}{\delta_{0}},
$$

being $\tau$ the average time it takes the two particles separated by an initial distance $\delta_{0}$ to reach a separation of $\delta_{f}$. In FTLE, one has to fix a finite integration time while in FSLE the initial and final distance between two adjacent particles $\left(\delta_{0}, \delta_{f}\right)$ are fixed. This two magnitudes have to be equivalent if they are measured with the corresponding time $T$ and distance $\delta_{f}$. Both exponents can be integrated forward and backward in time providing information about the barriers, boundaries or lines of strong stretching, by means of LCS. Under a transport perspective these LCS provide information about those areas where trajectories of initially close particles are quickly separated or particles of different origin attracted.

\footnotetext{
${ }^{1}$ IMEDEA (CSIC-UIB), Spain

${ }^{2}$ ETS Caminos Canales y Puertos (UCLM), Spain

${ }^{3} \mathrm{ICM}$ (CSIC), Spain

${ }^{4}$ IMEDEA (CSIC-UIB), Spain
} 
In this work we analyze the influence of waves on the modification on the LCS in a coastal area. FSLE are derived from ocean circulation model as well as from wave model and results are compared with available data from drifters.

\section{DATA AND METHODS}

The study has been performed in a semi-enclosed bay located in the southern side of the Island of Mallorca, Western Mediterranean Sea (Figure 1). Velocity data was obtained from the Regional Ocean Model System (ROMS), a free-surface, hydrostatic, primitive equation ocean model that uses stretched, terrainfollowing coordinates in the vertical and orthogonal coordinates in the horizontal (Song and Haidvogel, 1994).

Three different domains were implemented in order to obtain high resolution currents in the area of study. The coarser mesh with a resolution of $d \theta=d \lambda=1 / 74^{\circ}$ (e.g. $d x \simeq d y=1500 \mathrm{~m}$ ) take boundary conditions from an operational general ocean circulation model (MFSTEP). This domain, is nested to a second domain with a mesh of $d x=d y=300 \mathrm{~m}$ and the later to a third domain covering the study area which has a grid resolution of $d x=d y=75 \mathrm{~m}$ (Figure 1, right). This area is around $18 \mathrm{~km}$ wide with depths at its open boundary around $80 \mathrm{~m}$. The Bay is open to southerly and to southwesterly swells. The final grid is $348 \times 260$ nodes with 10 vertical levels. All domains were forced using wind provided by the PSU/NCAR mesoscale model MM5 (Grell et al., 1995).
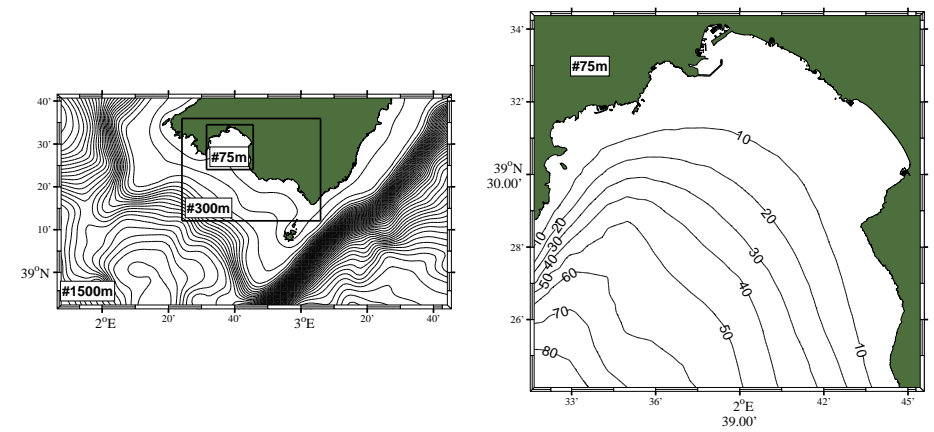

Figure 1: Area covering the domains for the wave and current models (left). Fine resolution grid detail (right).

The study was done from November $10^{\text {th }}$ to November $24^{\text {th }}, 2009$. During this period two different simulations were performed by forcing the ocean model with realistic wind fields (hereinafter set I) and forcing the model with the same wind fields plus the additional gradients of the radiation stress tensor (hereinafter set II). Temperature and salinity vertical structure was obtained from Levitus database (Locarnini et al., 2006; Antonov et al., 2006). The radiation stresses were obtained in the same domains of the circulation model by integrating WAM model, a third generation spectral wave model specifically designed for global and shelf sea applications (Komen et al., 1994). Boundary conditions for the first domain were taken from the operational model for the Western Mediterranean operated by the Spanish Harbor Authority. Although the wave model is not appropriate for very shallow waters (typical shallow water processes, e.g., diffraction, triad-wave interactions or depth-induced wave breaking are not considered), the model provides for typical wavelengths the correct wave field for depths higher than $10 \mathrm{~m}$.

For set II, the effects of waves are included in the three domains by adding the radiation stresses gradient as an additional forcing term acting on the surface. Stresses are updated every three hours since it is a reasonable interval to characterize the wave climate. For both simulations, velocity fields were stored every 5 minutes.

On November $19^{\text {th }}$ eight drifting buoys were deployed in the area of study for three days. The buoys were specifically designed for coastal studies and provided the position through a GPS position via GSM transmission every 5 minutes. Drifters were deployed at the vertex of a square in groups of 2 buoys. Deep water significant wave heights during this period reach $1 \mathrm{~m}$ from the southwest. 


\section{RESULTS}

We have performed simulations with the circulation model for the period considered storing surface velocity fields every 5 minutes. For both set I and set II the wind forcing and waves were updated every 3 hours. A grid of particles is launched every 3 hours for all the period. Particles are advected using a first order Euler algorithm which integrate velocity data from the numerical model in time. The FSLE at each point is defined as the maximum Lyapunov exponent of the 4 neighboring particles. On the other hand, the FTLE is computed using the spatial gradient of the flow at the four closest neighbors once the final position of each particle at the desired $T$ has been reached (Shadden et al., 2005).

LCS are dynamical patterns organizing the flow which can not be crossed by particles (Joseph and Legras, 2002). Besides, LCS obtained from Lyapunov exponents integrated forward in time, characterize repelling material lines (unstable structures) while LCS provided by Lyapunov exponents integrated backward in time, identify lines of attracting material (stable structures) (d'Ovidio et al., 2004). Intersection of LCS forward and LCS backward in time, correspond to hyperbolic points where stable and unstable directions of particles coexist.

To show the general behavior of the Lyapunov exponents we performed for a given day the FTLE and FSLE forward and backward in time using the numerical simulations of set I. The FTLE for November $23^{r d}, 2009$ at $18.00 \mathrm{~h}$ computed for time integration of $T=3 \mathrm{~h}, T=6 \mathrm{~h}, T=12 \mathrm{~h}$ and $T=24 \mathrm{~h}$ are shown in Figure 2 (top panels) where only the LCS defined as the maximum values of the FTLE are displayed. Structures with positive values correspond to FTLE integrated forward in time (hereinafter FTLEf) while structures with negative values to those exponents computed backward in time (hereinafter FTLEb). As seen when time integration in small, many LCS are present in the flow which are filtered when longer times are used. Intersections of LCS forward and LCS backward in time, correspond to hyperbolic points where stable and unstable directions of particles coexist.

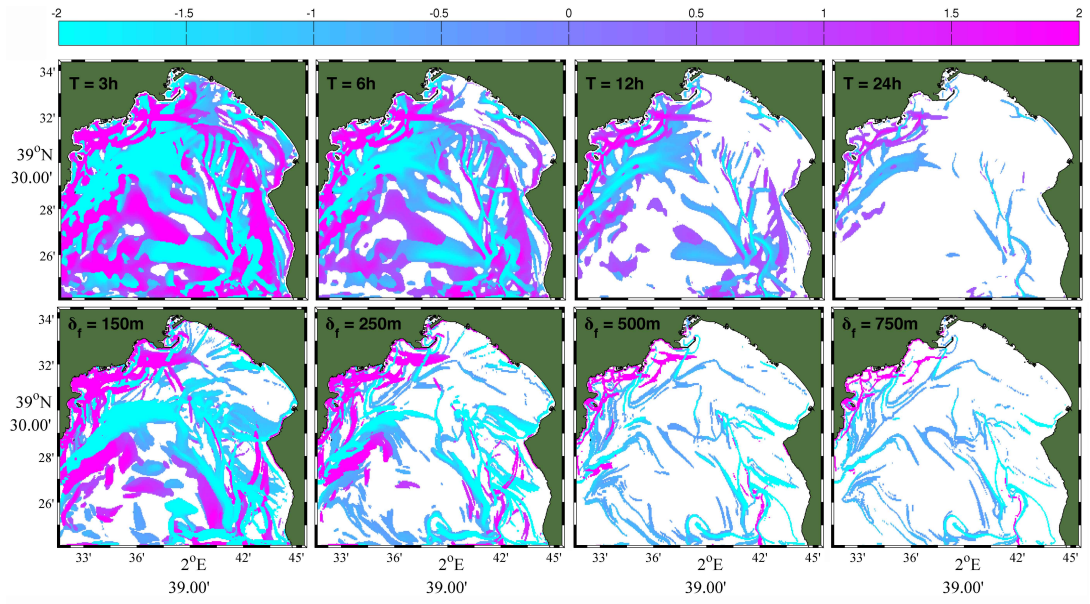

Figure 2: Finite Time Lyapunov Exponents for time integration $T=3 \mathrm{~h}, T=6 \mathrm{~h}, T=12 \mathrm{~h}$ and $T=24 \mathrm{~h}$ (top panel). Finite Size Lyapunov Exponents for final separation of $\delta_{f}=150 \mathrm{~m}, \delta_{f}=250 \mathrm{~m}, \delta_{f}=500 \mathrm{~m}$ and $\delta_{f}=750 \mathrm{~m}$ (bottom panel) (November $23^{r d}, 2009 ; 18.00 \mathrm{~h}$ ). Positive values of the exponents corresponds to forward integration and negative values to backward integration. FSLE units in day ${ }^{-1}$

Likewise, the FSLE computed for the final separation of particles of $\delta_{f}=150 \mathrm{~m}, \delta_{f}=250 \mathrm{~m}$, $\delta_{f}=500 \mathrm{~m}$ and $\delta_{f}=750 \mathrm{~m}$ are shown in Figure 2 (bottom panels) starting at November $23^{r d}$ at $18.00 \mathrm{~h}$. Similarly the LCS of the FSLE with positive values correspond to those exponents computed forward in time (hereinafter FSLEf) and those with negative values to exponents computed backward in time (hereinafter FSLEb). As seen, FTLE for time integration $T=12 \mathrm{~h}$ present a pattern of LCS similar to FSLE integrated for final separation of $\delta_{f}=250 \mathrm{~m}$. Maximum values for the Lyapunov exponent are of the order of 2 day $^{-1}$ corresponding to mixing times of $12 \mathrm{~h}$. It is noticeable that LCS from FSLE are more defined than those obtained by FTLE since for the latter, all particles of the domain will have a finite Lyapunov exponent but only those particles that reach the final $\delta_{f}$ will have a value of the Lyapunov exponent when FSLE are computed. Since we are interested in the mixing and presence of barriers of transport induced by 
waves, we will show only the LCS obtained from the FSLEb. The final distance considered is $\delta_{f}=250 \mathrm{~m}$. which is roughly 3 mesh points.

The influence of waves in the stirring of the surface layer in the study area is assessed by analyzing the LCS from periods with different wave conditions. Starting on November $15^{\text {th }}$ relatively mild wave conditions were present in the area. For this period, we compute the LCS of the FSLEb for both set I (Figure 3, left panel) and for set II (Figure 3, right panel). LCS are shown for November $15^{\text {th }}$ to November $18^{\text {th }}$ at $00.00 \mathrm{~h}$. Not surprisingly, the structure of the LCS display a similar pattern for both datasets in this 4-days analyzed. A barrier parallel to the coast is almost present during all the period separating two areas in the shallow zone. Shallow areas are characterized by stronger mixing as seen by the complex pattern of the LCS. Mean values of the FSLEb for currents are $-0.13,-0.17,-0.31$ and -0.31 day $^{-1}$ for November $15^{\text {th }}, 16^{\text {th }}, 17^{\text {th }}$ and $18^{\text {th }}$ respectively. For the same period these values for waves and currents are -0.18 , $-0.17,-0.29$ and -0.31 day $^{-1}$.

To show the utility of LCS in the study of the dispersion of surface material such as pollutants as well as the indication of such structures to be zones of attracting flow, we deployed 296 virtual neutral particles at four different locations. For the period comprised between November $15^{\text {th }}-18^{\text {th }}$, the path followed and the general behaviour of lagrangian particles are similar for only wind, and wind and waves conditions. It is noticeable that those particles located over LCS remain there and those particles located at different sides of the structures do not cross the LCS tending to the more attractive structures. LCS provide therefore a dynamical picture of the organization of the flow that is a powerful tool when analyzing areas of strong convergence (divergence) of the flow.

A different situation is obtained from November $21^{s t}$ to November $24^{\text {th }}$. During this period, significant wave height $\left(H_{s}\right)$ measured at deep waters reached $1 \mathrm{~m}$. The effect of waves is to increase mixing and, therefore, to modify the transport at the surface. The LCS for this period are displayed in Figure 4 for set I (left) and for set II (right). LCS are displayed each day at $00.00 \mathrm{~h}$. The mean value for the wind stress during this period is $0.1 \mathrm{~N} / \mathrm{m}^{2}$ which is increased by the effects of waves up to $0.25 \mathrm{~N} / \mathrm{m}^{2}$. The barrier that was located near the coast in mild wave conditions is moved onshore appearing new areas of strong mixing in the middle of the Bay.

Contrarily to the previous situation, the LCS from set I and the LCS from set II show a very different pattern. In general, the effect of waves is to break the LCS generating chaotic tangles that increase the horizontal stirring and mixing. The mean values of the FSLE for set I are $-0.27,-0.31,-0.27$ and -0.40 days $^{-1}$ for currents at November $21^{\text {th }}, 22^{\text {th }}, 23^{\text {th }}$ and $24^{\text {th }}$ respectively, being $-0.31,-0.33,-0.30$ and -0.42 days $^{-1}$ for set II. Those data suggest that in this analized case the effects of waves is to increase mixing around a $10 \%$ respect the simulations made only with wind forcing.

Again, we deployed 296 virtual particles at four different areas selected so as to be at both sides of the LCS of the initial day. On November $22^{\text {nd }}$ particles launched at the flow from set I are located on areas characterized by low values of FSLEb implying small dispersion (Figure 4, left). The initial shape of the particles deployed are maintained after 24 hours. Nevertheless, particles within the flow driven by set II are distributed in deep waters over a LCS that is the result of the waves that broke the original shape (Figure 4, right). Moreover two different sets collapsed to this line and finally got mixed (see diamonds and stars on Figure 4). One day after, on November $23^{r d}$, differences become more important. The two sets of particles originally deployed at the East, have been moved to the center of the Bay since they have been attracted by the LCS which cross the bay in a south-north direction. At evening on November $23^{\text {th }}$ wave energy decrease which can be inferred from the LCS displayed on the $24^{\text {th }}$. Both LCS fields present the same structure of the manifolds (Figure 4, bottom). However, due to the history of the dynamics, particles are disposed over different lines of attraction being particles from different sets totally mixed in set II (see circles, diamonds, squares and star at Figure 4, bottom, left and right).

To elucidate the role of the radiation stress from waves on the computation of LCS in coastal areas, eight lagrangian drifters were deployed on November $19^{\text {th }}$ at 12.00. Figure 5 and Figure 6 display the LCS every 6 hours, starting the date of the deployment for set I and for set II, respectively, and trajectories followed by the buoys. The first 18 hours waves were below $30 \mathrm{~cm}$ and it is reflected in the pattern of the LCS. Along this period, there is a clear separation between two areas of the Bay being the deeper part more diffusive than the shallow part due to the fact that dynamics within the Bay is mainly driven by shelf slope dynamics and relatively mild small scale wind driven circulation acts in the Bay. Drifters at the shallow part travel small distances indicating high residence time of waters for this period. These buoys fall in small 


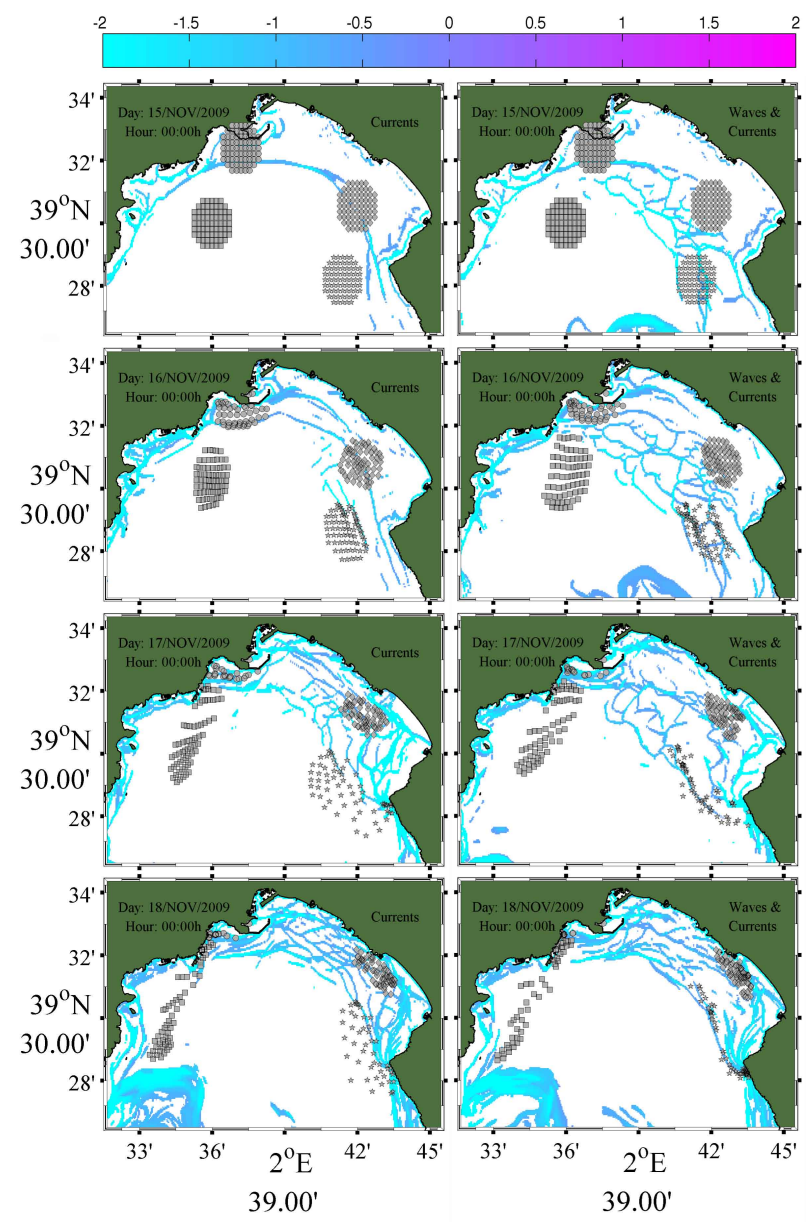

Figure 3: Daily snapshots of Finite Size Lyapunov Exponents backward in time for a final separation of $\delta_{f}=250 \mathrm{~m}$ computed by currents (left panel) and with waves and currents (right panel) starting on November $15^{\text {th }}$. Virtual particles at four different areas are marked by circles, diamonds, squares and stars. FSLE units in day $^{-1}$

diffusive areas in both, set I (Figure 5) and set II (Figure 6). Drifters deployed at deeper waters, are attracted by the strong structure that cross the Bay form East to West moving along it. On November $20^{\text {th }}$ at 6.00 $H_{s}$ increased and differences between the two patterns of LCS became evident. A new line of attraction appeared for the set II parallel to the main structure. This line is fully developed on November $20^{\text {th }}$ at 18.00 not being present when computing the LCS in absence of waves (Figure 5). This new structure continues evolving and moving onshore increasing mixing in set II. The path of the lagrangian drifters follow the LCS from FSLEb provided by set II.

\section{CONCLUSION}

We have shown that either FTLE and FSLE can be used by choosing the suitable scale to analyze the dynamical field of coastal areas. The LCS integrated backward in time, provide valuable information when trying to response and mitigate possible spills in these areas. However, we have seen that a proper characterization of currents including the effects induced by waves is necessary in order to obtain a correct description of the mixing activity and the coherent structures that control the transport at the scale of interest.

\section{ACKNOWLEDGEMENTS}

Authors would like to thank financial support from Spanish MICINN thought projects CTM201016915 and CGL2011-22964 and from Med Project TOSCA (G-MED09-425). 


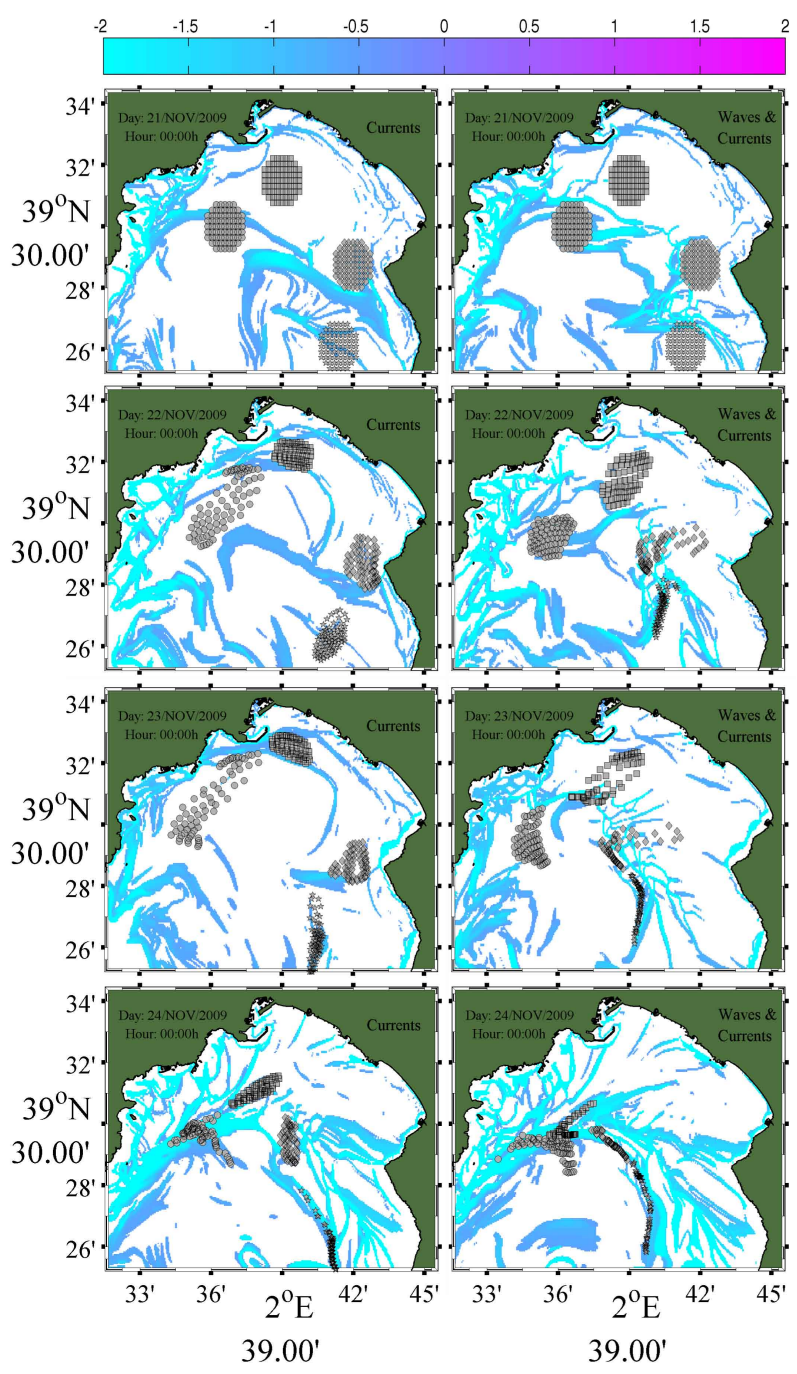

Figure 4: Daily snapshots of FSLEb for a final separation of $\delta_{f}=250 \mathrm{~m}$ computed by currents (left panel) and with waves and currents (right panel) starting on November $21^{\text {th }}$. Virtual particles at four different areas are marked by circles, diamonds, squares and stars. FSLE units in day ${ }^{-1}$

\section{References}

Antonov, J. I., R. A. Locarnini, T. P. Boyer, A. V. Mishonov, and H. E. Garcia (2006), World Ocean Atlas 2005, Volume 2: Salinity. S. Levitus, Ed. NOAA Atlas NESDIS 62, U.S. Government Printing Office, Washington, D.C., 182 pp.

d'Ovidio, F., V. Fernandez, E. Hernandez-Garcia and C. Lopez (2004), Mixing structures in the Mediterranean sea from Finite-Size Lyapunov Exponents, Geophys. Res. Lett, 31, L17203, doi:10.1029/2004GL020328.

Galan, A., Orfila, A., Simarro, G., Hernandez-Carrasco, I. and Lopez, C. (2012), Wave mixing rise inferred from Lyapunov exponents, Env. Fluid Mech., 12 (3), 291-300, doi: 0.1007/s10652-012-9238-3.

Grell, G. A., J. Dudhia, and D. R. Stauffer (1995), A description of the fifth-generation penn state/NCAR mesoscale model (MM5), NCAR/TN-398+STR, National Center for Atmospheric Research, Boulder, $\mathrm{CO}, 122 \mathrm{pp}$.

Haller, G. and G. Yuan (2000), Lagrangian coherent structures and mixing in two-dimensional turbulence, 
Physica D, 147, 352-370. How reliable are Finite-Size Lyapunov Exponents for the assessment of ocean dynamics.

Hernandez-Carrasco, I., C. Lopez, E. Hernandez-Garcia and A. Turiel (2011), Ocean Modelling,36, 208218.

Joseph, B. and B. Legras (2002), Relation between kinematic boundaries, stirring, and barriers for the Antartic Polar vortex, Journal of the Atmospheric Sciences, 59, 1198-1212.

Komen, G.J., L. Cavaleri, M. Donelan, K. Hasselmann, S. Hasselmann and P.A.E.M. Janssen (1994), Dynamics and Modelling of Ocean Waves, Cambridge University Press.

Lapeyre, G. (2002), Characterization of finite-time Lyapunov exponents and vectors in two-dimensional turbulence, Chaos, 12 (3), 688-698, doi:10.1063/1.1499395.

Locarnini, R. A., A. V. Mishonov, J. I. Antonov, T. P. Boyer, and H. E. Garcia (2006), World Ocean Atlas 2005, Volume 1: Temperature. S. Levitus, Ed. NOAA Atlas NESDIS 61, U.S. Government Printing Office, Washington, D.C., 182 pp.

Longuet-Higgins, M.S. and R.W. Stewart (1964), Radiation stresses in water waves: a physical discussion

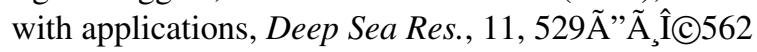

Mancho, M., E. Hernandez-Garcia, D. Small, S. Wiggins and V. Fernandez (2008), Lagrangian Transport through an Ocean Front in the Northwestern Mediterranean Sea, J. of Phys. Ocean., 38, 1222-1237.

Molcard, A.,A.C. Poje, and T.M. Özgökmen, (2006), Directed drifter launch strategies for Lagrangian data assimilation using hyperbolic trajectories, Ocean Modelling, 12, 268-289.

Özgökmen, T.M., A. Griffa, A.J. Mariano and L.I. Piterbarg (2000), On the predictability of Lagrangian trajectories in the ocean, J. Atmos. Ocean. Tech., 17 (3), 366-383.

Shadden,S. C., F. Lekien and J. E. Marsden (2005), Definition and properties of Lagrangian coherent structures from finite-time Lyapunov exponents in two-dimensional aperiodic floows, Physica D, 212, (3-4), 271-304.

Song, Y. and D. B. Haidvogel (1994), A semi-implicit ocean circulation model using a generalized topography-following coordinate system, J. Comp. Phys., 115 (1), 228-244.

Wiggins, S. (1992), Chaotic transport in Dynamical Systems, Springer Verlag, New York. 


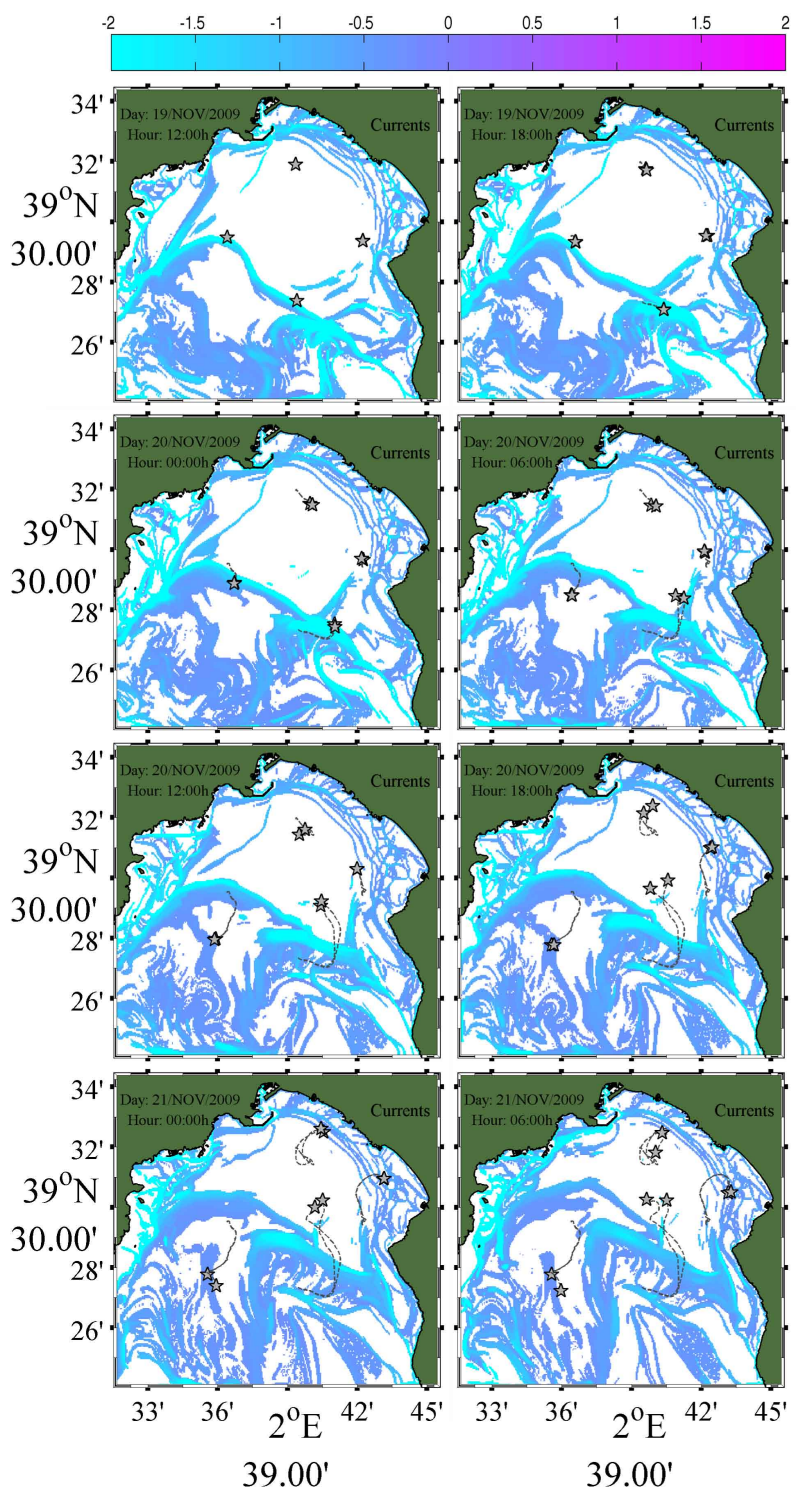

Figure 5: 6 hour snapshot of FSLEb for a final separation of $\delta_{f}=250 \mathrm{~m}$ computed by currents and trajectories of lagrangian drifters. FSLE units in day $^{-1}$ 


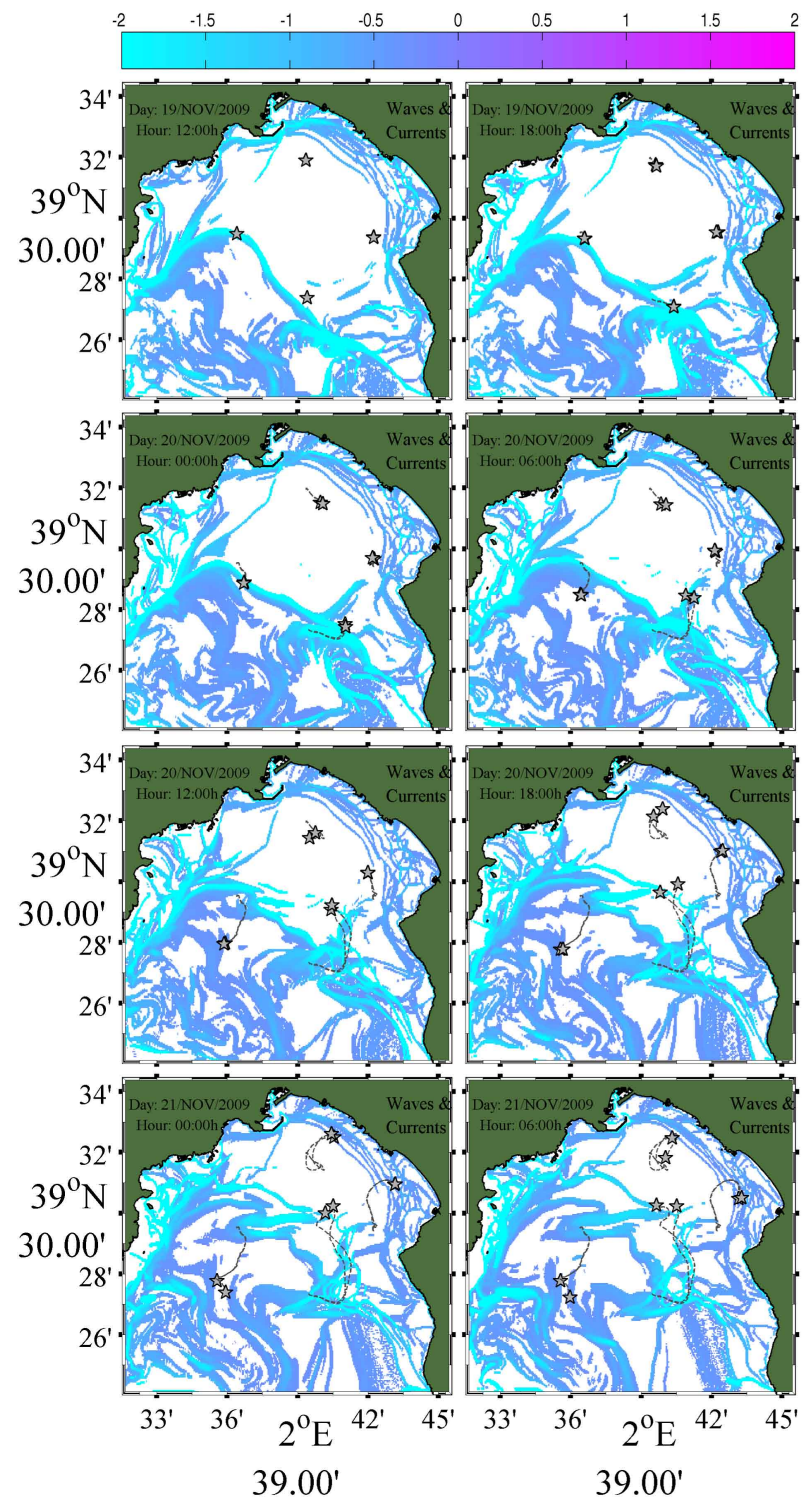

Figure 6: 6 hour snapshot of FSLEb for a final separation of $\delta_{f}=250 \mathrm{~m}$ computed by currents and waves and trajectories of lagrangian drifters. FSLE units in day $^{-1}$ 